\title{
A novel protein expression signature differentiates benign lipomas from well-differentiated liposarcomas
}

\author{
QUANG MATHER $^{1 *}$, JONATHON PRIEGO $^{1 *}$, KRISTI WARD $^{1 *}$, VERMA KUNDAN $^{1}$, \\ DAT TRAN $^{1}$, ALOK DWIVEDI ${ }^{1,2}$ and BRAD A. BRYAN ${ }^{1}$ \\ ${ }^{1}$ Department of Biomedical Sciences and ${ }^{2}$ Division of Biostatistics and Epidemiology, Paul L. Foster School \\ of Medicine, Texas Tech University Health Sciences Center, El Paso, TX 79905, USA
}

Received May 12, 2017; Accepted June 13, 2017

DOI: $10.3892 /$ mco.2017.1325

\begin{abstract}
Benign lipomas and well-differentiated liposarcomas share many histological and molecular features. Due to their similarities, patients with these lipomatous tumors are misdiagnosed up to $40 \%$ of the time following radiological detection, up to $17 \%$ of the time following histological examination, and in as many as $15 \%$ of cases following fluorescent in situ hybridization for chromosomal anomalies. Incorrect classification of these two tumor types leads to increased costs to the patient and delayed accurate diagnoses. In this study, we used genomics analysis to identify several genes whose mRNA expression patterns were significantly altered between lipomas and well-differentiated liposarcomas. We confirmed our findings at the protein level using a panel of 30 human lipomatous tumors, revealing that C4BPB, class II, major histocompatibility complex, CIITA, EPHB2, HOXB7, GLS2, RBBP5, and regulator of RGS2 protein levels were increased in well-differentiated liposarcomas compared to lipomas. We developed a multi-protein model of these markers to increase discriminatory ability, finding the combined expression model with CIITA and RGS2 provided a high ability $(\mathrm{AUC}=0.93)$ to differentiate between lipomas and well-differentiated liposarcomas with sensitivity at $83.3 \%$ and specificity at $90.9 \%$.
\end{abstract}

\section{Introduction}

Lipomatous tumors are a highly diverse group of mesenchymal neoplasms characterized by an overgrowth of adipose cells or their precursors. Benign lipomas are the most prevalent type

Correspondence to: Dr Brad A. Bryan, Department of Biomedical Sciences, Paul L. Foster School of Medicine, Texas Tech University Health Sciences Center, MSB1 Room 2111, 5001 El Paso Drive El Paso, TX 79905, USA

E-mail: brad.bryan@ttuhsc.edu

*Contributed equally

Key words: liposarcoma, lipoma, biomarker, diagnostic, immunohistochemistry, genomics of lipomatous tumor and are the most common soft tissue tumor (1). Lipomas affect approximately $1 \%$ of the general population, appearing most often in patients aged 40-60 years and generally ranging in size from 1 to $3 \mathrm{~cm}$, although rare giant lipomas can grow to $20 \mathrm{~cm}$ in diameter and weigh up to $5 \mathrm{~kg}(2,3)$. These relatively common tumors are rarely life threatening and in most cases do not require medical treatment unless the tumor restricts movement or causes pain. By contrast, malignant liposarcomas are much more rare (approximately 2.5 cases per million individuals), but rank as the second most common of all soft tissue sarcomas in humans (4). Liposarcomas can be characterized into multiple, phenotypically diverse subtypes including well-differentiated, myxoid/round cell liposarcomas, pleiomorphic, and de-differentiated. These malignant tumors exhibit 5-year survival rates as low as 39\% depending on the particular histological subtype (5).

Many molecular and clinical similarities exist between lipomas and low-grade liposarcomas such as the well-differentiated subtype $(6,7)$. Thus, a diagnostic dilemma can occur with regard to differentiating these tumors. When comparing lipomas and low-grade liposarcomas, patients are misdiagnosed $30-40 \%$ of the time following radiological detection (8-10) and in 7-17\% of histological evaluations (11). Even fluorescent in situ hybridization (FISH) for MDM2-CDK4 amplification (considered the gold standard for distinguishing lipomas from low-grade and de-differentiated liposarcomas) is inaccurate and/or provides uninterpretable results in 10-15\% of cases (12-16). Diagnostic accuracy is also problematical due to morphological heterogeneity within particular lipomatous tumors. For instance, benign pleomorphic lipomas exhibit unusual features such as the presence of hibernomas or tumor pleomorphism that can lead to confusion with malignant liposarcomas (17). Many liposarcomas display transitional features of low- to high-grade lesions or consist of well-differentiated regions associated with non-lipogenic sarcoma often resembling malignant fibrous histiocytomas or fibrosarcoma (17). Further complicating this issue is a recent study that reveals MDM2 and CDK4 genes are both amplified in up to $5 \%$ of large and deep-seated lipomas, suggesting that the clinical significance of gene amplifications for lipomatous tumors is unclear and requires further studies (18).

Accurate, timely, and cost effective diagnosis of lipomatous tumors is essential for proper patient treatment and increased 
long-term survival. In the present study, we utilized a combination of bioinformatics and immunohistochemistry (IHC) analysis to accurately and sensitively identify biomarkers that effectively distinguish benign from low-grade malignant lipomatous tumors.

\section{Materials and methods}

Meta-analysis of lipomatous tumor gene expression. A whole genome RNA expression dataset [Gene Expression Omnibus (GEO) reference series GSE6481, deposited by Nakayama et al (14)] was analyzed to identify gene expression alterations between benign lipomas and well-differentiated liposarcomas. This dataset compared the global gene expression profiles of various soft tissue sarcomas including lipomas $(n=3)$ and well-differentiated liposarcomas $(n=3)$. Tab-delimited files from these data were input into Cluster 3.0, normalized, and clustered using a correlation (uncentered) similarity metric with a centroid linkage. Data were visualized using Java Treeview.

Case material. Tissue arrays of formalin-fixed, paraffinembedded lipomatous tumor blocks were obtained from Super Bio Chips (Seoul, Korea) (www.tissue-array.com). These clinically characterized tumor samples consisted of 2-mm diameter cores with a section thickness of 4 microns and were composed of lipomas $(n=11)$ and well-differentiated liposarcomas $(n=20)$. The cases were blindly reviewed and confirmed by a pathologist.

IHC. Sections were deparaffinized, rehydrated, and treated for antigen retrieval using Trilogy (cat. no. 920P-10; Cell Marque, Rocklin, CA, USA). To block non-specific binding, the sections were incubated in background block solution (cat. no. 927B-05; Cell Marque) at room temperature for $10 \mathrm{~min}$ before application of primary antibody. Antibodies used in this study included: ATP-binding cassette, sub-family B member 11 (ABCB11) (cat. no. ab155421), bone morphogenetic protein 8A (BMP8A) (cat. no. ab60290) and complement component 4 binding protein $\beta$ (C4BPB) (cat. no. ab105507) (all from Abcam, Cambridge, MA, USA), class II, major histocompatibility complex, transactivator (CIITA) (cat. no. sc48797; Santa Cruz Biotechnology, Inc., Santa Cruz, CA, USA), ephrin receptor B2 (EPHB2) (cat. no. ab150652), glutaminase 2 (GLS2) (cat. no. ab113509), homeobox B7 (HOXB7) (cat. no. ab111018), protein kinase N2 (PKN2) (cat. no. ab32395), RAB6B, member RAS oncogene family (RAB6B) (cat. no. ab55660), retinoblastoma-binding protein 5 (RBBP5) (cat. no. ab84511), serine incorporator 2 (SERINC2) (cat. no. ab134312), G-protein signaling 2 (RGS2) (cat. no. ab36561) $\gamma$-synuclein (SNCG) (cat. no. ab55424), sex determining region Y-box 30 (SOX-30) (cat. no. ab26024), and thyroid-stimulating hormone receptor (TSHR) (cat. no. ab27974) (all from Abcam). The sections were stained using the HRP/DAB Detection IHC kit (cat. no. ab80436; Abcam) and counterstained with hematoxylin.

Quantification of and statistical analysis of IHC. Immunopositivity was scored semi-quantitatively for the percentage of tumor cell staining ( 0 , negative; 1 , weak staining; 2 , moderate staining; 3 , strong) and intensity ( 0 , negative; $1,<25 \%$ of tumor cells stained; 2, 25-49\% of tumor cells stained; $3,50-74 \%$ of tumor cells stained; $4,>75 \%$ of tumor cells stained). The product of the percentage staining and intensity values represented the IHC score for each tumor. Mean IHC values (mean \pm SEM) were calculated using the Mann-Whitney ranksum test. A logistic regression model was developed for each protein to test its ability to differentiate between the two tumor types. The model's discriminatory performance was assessed using area under the curve (AUC) with a 95\% confidence interval. Furthermore, the threshold for each marker and combined model in differentiating lipoma from well-differentiated liposarcoma was determined using receiver operating characteristics analysis. The cut-off value was chosen where sensitivity and specificity were found to be similar.

\section{Results}

Nakayama et al (19) previously published data comparing the relative global gene expression profiles of various soft tissue sarcomas. These data are freely and publically available in the GEO (no. GSE6481, www.ncbi.nlm.nih.gov/geo/). Included in this genomic dataset are transcriptional profiles from benign lipomas and well-differentiated liposarcomas. The data from this analysis can serve as a guide for further validation experiments using larger patient datasets to identify biomarkers that can differentiate lipomas from well-differentiated liposarcomas. Unsupervised hierarchical clustering analysis of the lipoma and well-differentiated liposarcoma gene expression data from Nakayama et al (14) showed that a marked divergence in expression profiles occurred even within each tumor subtype. However, clear clustering occurred for the tumors based on their histological type (Fig. 1). This result suggests that despite the current clinical difficulty in distinguishing these tumor types based on radiology, histology, or cytogenetics, differences in the gene expression profiles may be identified and utilized to differentiate lipomas from well-differentiated liposarcomas.

We selected 15 genes from this meta-analysis that were differentially regulated in well-differentiated liposarcomas compared to lipomas for further investigation. The genes included $A B C B 11, B M P 8 A, C 4 B P B, C I I T A, E P H B 2, G L S 2$, HOXB7, PKN2, RAB6B, RBBP5, RGS2, SERINC2, SNCG, $S O X 30$ and TSHR. We validated the expression of the 15 genes at the protein level using IHC on an independent panel of clinically defined lipomatous tumors. This tumor panel consisted of 11 lipoma tumors and 19 well-differentiated liposarcomas. The clinico-pathological characteristics of this patient dataset are reported in Table I. The distribution of age, sex, and location were almost similar between the groups. Semi-quantitative analysis of the staining across the tumor panel demonstrated that C4BPB, CIITA, EPHB2, HOXB7, GLS2, RBBP5, and RGS2 protein levels were increased in well-differentiated liposarcomas compared to lipomas (Figs. 2 and 3). Despite changes in gene expression observed in our meta-analysis, the IHC analysis did not detect differences in the protein expression of ABCB11, BMP8A, PKN2, RAB6B, SERINC2, SNCG, SOX30, or TSHR between lipomas and well-differentiated liposarcomas (data not shown).

To confirm the statistical significance of our findings, logistical regression models we utilized to assess the individual discriminatory value of each protein to distinguish between 


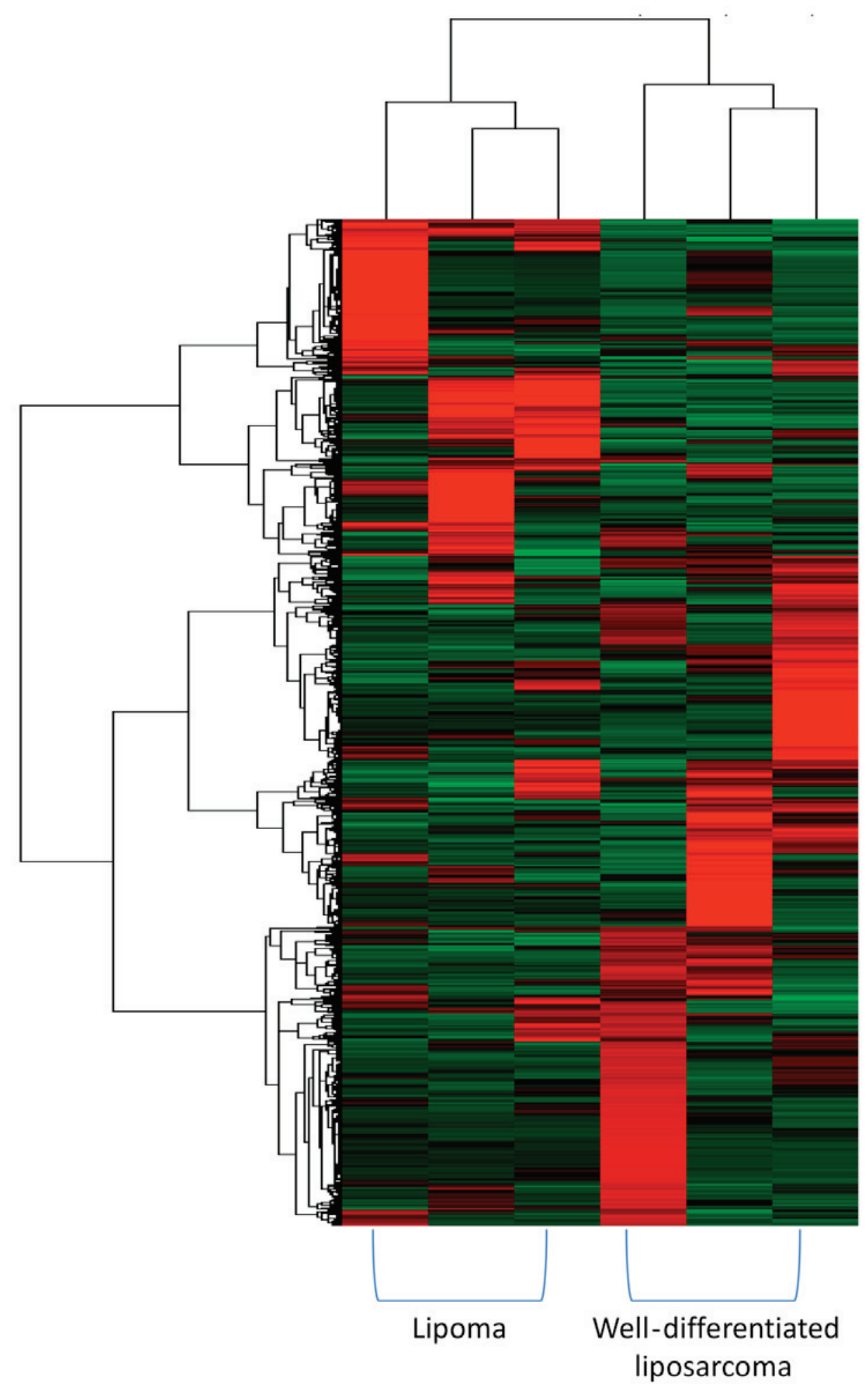

Figure 1. Unsupervised hierarchical clustering analysis. Gene expression profiles of three benign lipomas and three well-differentiated liposarcomas from the publically available sarcoma gene expression dataset (GEO no. GSE6481) is displayed as a heat map. Individual genes are represented along the vertical axis, and lipomas $(\mathrm{N}=3)$ and well-differentiated liposarcomas $(\mathrm{N}=3)$ are represented along the horizontal axis. Red, overexpression; green, underexpression. GEO, Gene Expression Omnibus.

lipoma and well-differentiated liposarcoma. Due to the large variation in IHC scores, a natural log transformation after adding 1 was made for each differentially expressed protein. Table II reveals the individual model of each marker for differentiating well-differentiated liposarcoma from lipoma. These individual models can be used to differentiate two types of tumor groups, and all considered markers were found to be significantly associated with well-differentiated liposarcoma. In the unadjusted analysis, the highest strength of association with well-differentiated liposarcoma as compared with lipoma was observed for HOXB7 (OR=20.590) followed by CIITA $(\mathrm{OR}=14.963)$ and $\mathrm{RGS} 2(\mathrm{OR}=11.509)$. Table III shows the 
Table I. Patient characteristics.

WD

Variables

Overall Lipoma liposarcoma

No. of patient samples

$\begin{array}{ccc}30 & 11 & 19 \\ 51 \pm 13 & 50 \pm 10 & 51 \pm 15 \\ 51(60) & 53(30) & 49(60) \\ 21 / 9 & 8 / 3 & 13 / 6\end{array}$

Age, years (mean \pm SD)

Age (median years)

$\operatorname{Sex}(\mathrm{F} / \mathrm{M})$

Tumor location

(no. of tumors)

\begin{tabular}{llll} 
Axilla & 4 & 1 & 3 \\
Back & 2 & 2 & 0 \\
Breast & 2 & 2 & 0 \\
Buttock & 1 & 0 & 1 \\
Head and neck & 4 & 3 & 1 \\
Legs & 7 & 1 & 6 \\
Mediastinum & 1 & 0 & 1 \\
Mesentery & 1 & 0 & 1 \\
Retroperitoneum & 5 & 0 & 5 \\
Shoulder & 3 & 2 & 1 \\
\hline
\end{tabular}

WD, well-differentiated; F, female; $\mathrm{M}$, male.

Table II. Individual model for differentiating well differentiated liposarcoma from lipoma.

\begin{tabular}{crccc}
\hline Model & RC & $95 \%$ CI & P-value & OR \\
\hline C4BPB & 2.169 & $0.329,4.009$ & 0.021 & 8.751 \\
_cons & -2.347 & $-4.753,0.059$ & 0.056 & \\
EPHB2 & 1.672 & $0.030,3.314$ & 0.046 & 5.321 \\
_cons & -2.459 & $-5.423,0.505$ & 0.0505 & \\
GLS2 & 1.992 & $0.415,3.569$ & 0.013 & 7.331 \\
_cons & -3.278 & $-6.332,-0.223$ & 0.035 & \\
HOXB7 & 3.025 & $0.753,5.297$ & 0.009 & 20.590 \\
_cons & -5.135 & $-9.384,-0.886$ & 0.018 & \\
RBBP5 & 1.520 & $0.246,2.794$ & 0.019 & 4.572 \\
_cons & -2.001 & $-4.267,0.265$ & 0.084 & \\
RGS2 & 2.443 & $0.633,4.253$ & 0.008 & 11.509 \\
_cons & -2.393 & $-4.608,-0.179$ & 0.034 & \\
CIITA & 2.706 & $0.641,4.771$ & 0.010 & 14.963 \\
_cons & -2.320 & $-4.314,-0.326$ & 0.023 & \\
\hline
\end{tabular}

Note that all markers are natural log transformed after adding 1. RC, regression coefficient; $\mathrm{CI}$, confidence interval; $\mathrm{OR}$, odds ratio.

cut-off value of each marker to be used for differentiating the two groups. The highest classification accuracy was obtained using CIITA ( $\geq 2$ ) with sensitivity $77.8 \%$ and specificity $90.1 \%$ followed by RGS2 $(\geq 2)$ with sensitivity $66.7 \%$ and specificity $81.8 \%$ and GLS2 $(\geq 6)$ with sensitivity $72.2 \%$ and specificity $72.7 \%$. The overall performance of each model was reported in Table IV. The maximum AUC was obtained for the CIITA
Table III. Individual threshold of each marker for differentiating well-differentiated liposarcoma from lipoma.

\begin{tabular}{lcccc}
\hline & Se $(\%)$ & Sp $(\%)$ & $\begin{array}{c}\text { Correctly } \\
\text { classified }\end{array}$ & $\begin{array}{c}\text { LR+ } \\
\text { log threshold }\end{array}$ \\
\hline C4BPB & 66.7 & 63.6 & $65.5 \%, 1.83$ & $1.39(3)$ \\
EPHB2 & 61.1 & 63.6 & $62.1 \%, 1.68$ & $1.95(6)$ \\
GLS2 & 72.2 & 72.7 & $72.4 \%, 2.65$ & $1.95(6)$ \\
HOXB7 & 72.2 & 63.6 & $69.0 \%, 1.99$ & $1.95(6)$ \\
RBBP5 & 66.7 & 72.7 & $69.0 \%, 2.44$ & $1.95(6)$ \\
RGS2 & 66.7 & 81.8 & $72.4 \%, 3.67$ & $1.39(3)$ \\
CIITA & 77.8 & 90.1 & $82.8 \%, 8.56$ & $1.10(2)$ \\
\hline
\end{tabular}

Se, sensitivity; Sp, specificity; LR+, likelihood ratio positive.

Table IV. Overall performance of each marker in differentiating well differentiated liposarcoma from lipoma.

\begin{tabular}{lcc}
\hline Model & AUC & $95 \%$ CI \\
\hline C4BPB & 0.790 & $0.619,0.962$ \\
EPHB2 & 0.732 & $0.547,0.918$ \\
GLS2 & 0.798 & $0.625,0.971$ \\
HOXB7 & 0.828 & $0.676,0.980$ \\
RBBP5 & 0.803 & $0.641,0.965$ \\
RGS2 & 0.854 & $0.710,0.997$ \\
CIITA & 0.894 & $0.783,1.000$
\end{tabular}

AUC, area under the curve; CI, confidence interval.

Table V. Individual model for differentiating liposarcoma as compared to lipoma.

\begin{tabular}{lcccr}
\hline Model & RC & $95 \% \mathrm{Cl}$ & P-value & \multicolumn{1}{c}{ OR } \\
\hline Rbbp5 & 1.538 & $-0.300,3.377$ & 0.101 & 4.657 \\
Ciita & 2.784 & $0.496,5.072$ & 0.017 & 16.179 \\
_cons & -4.822 & $-9.022,-0.622$ & 0.024 & \\
\hline
\end{tabular}

$\mathrm{RC}$, regression coefficient; CI, confidence interval; OR, odds ratio.

model (0.894) followed by RGS2 (0.854). According to AUCs, the individual performances of C4BPB, GLS2, RBBP5 and HOXB7 models were found to be almost similar. The lowest AUC was obtained for EPHB2 model. This indicates that the individual CIITA model can be reliably used for differentiating the two types of lipomatous tumors followed by the RGS2 model.

We developed a multi-protein model of these markers to increase discriminatory ability. Our analysis revealed the combined model with Ciita and Rgs 2 provides the highest ability $(\mathrm{AUC}=0.93)$ to differentiate between lipomas and well-differentiated liposarcomas (Table V). The 


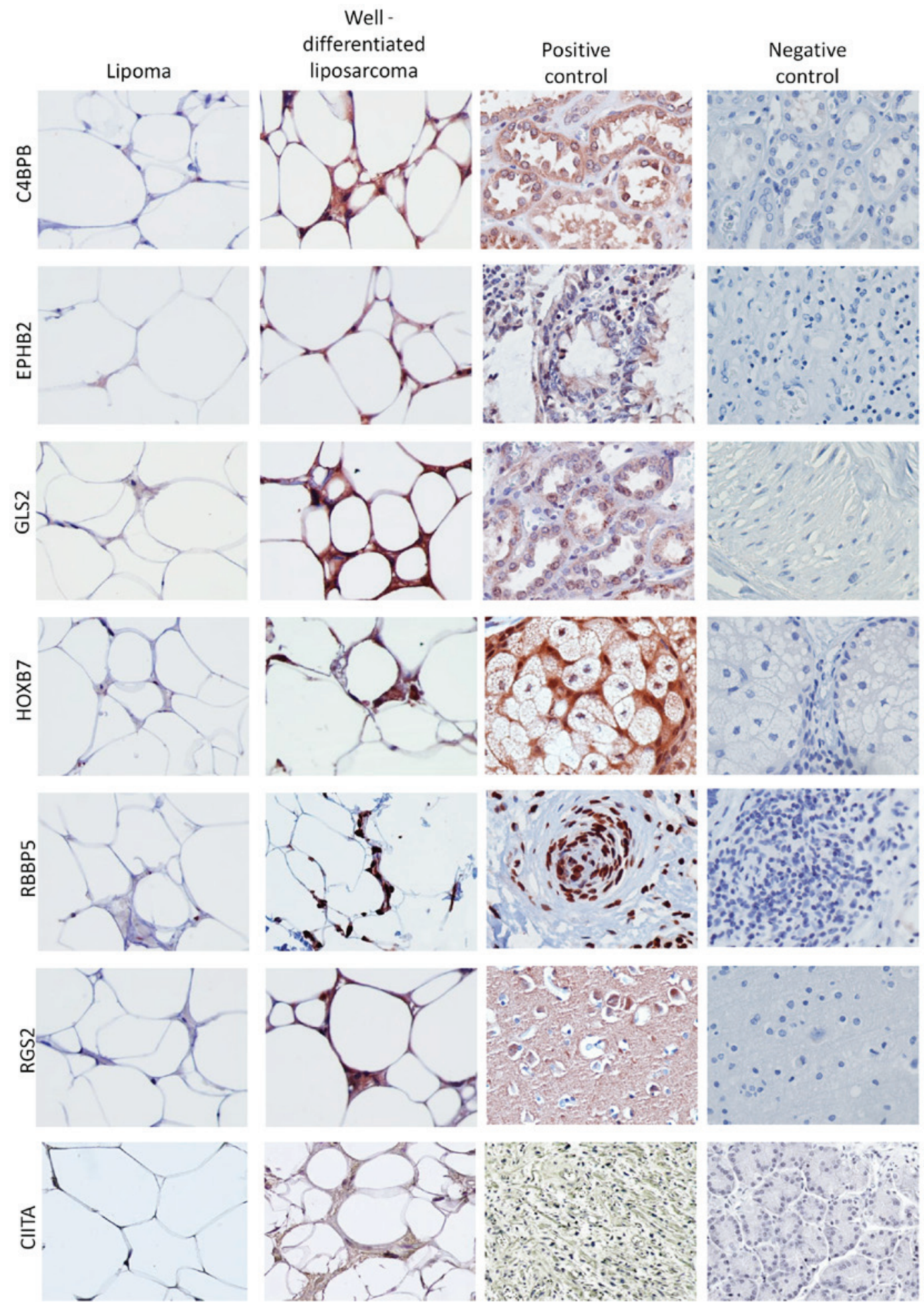

Figure 2. IHC of diagnostic markers. Representative IHC images for seven proteins whose expression was differentially regulated between benign lipomas and well-differentiated liposarcomas. Negative controls lacking the primary antibody and positive controls from the tissues shown by the Human Protein Atlas (www.proteinatlas.org) to be positive for each antigen were used to ensure immunopositivity was reliable. Brown staining indicates immunopositivity. IHC, immunohistochemistry.

following equation can be used for predicting probability of well-differentiated liposarcoma as compared to lipoma using Citta and Rgs2 IHC scores: $\mathrm{P}(\mathrm{WDL})=\exp [-4.822+(2.183$ $\left.\left.\mathrm{x} \log ^{\text {Ciita }}\right)+\left(1.404 \times \log ^{\mathrm{Rgs} 2}\right)\right] /\{1+\exp [-4.822+(2.183 \mathrm{x}$ $\left.\left.\left.\log ^{\text {Ciita }}\right)+\left(1.404 \times \log ^{\mathrm{Rgs} 2}\right)\right]\right\}$ where $\mathrm{P}(\mathrm{WDL})$ is the probability of the tumor being a well-differentiated liposarcoma, and Ciita and Rgs 2 are the IHC scores for each protein, respectively. The cut-off value for the combined model differentiated the two tumor types with sensitivity at $83.3 \%$ and specificity at $90.9 \%$.

\section{Discussion}

Differentiation between lipoma and well-differentiated liposarcoma can be challenging, particularly in core biopsies where 

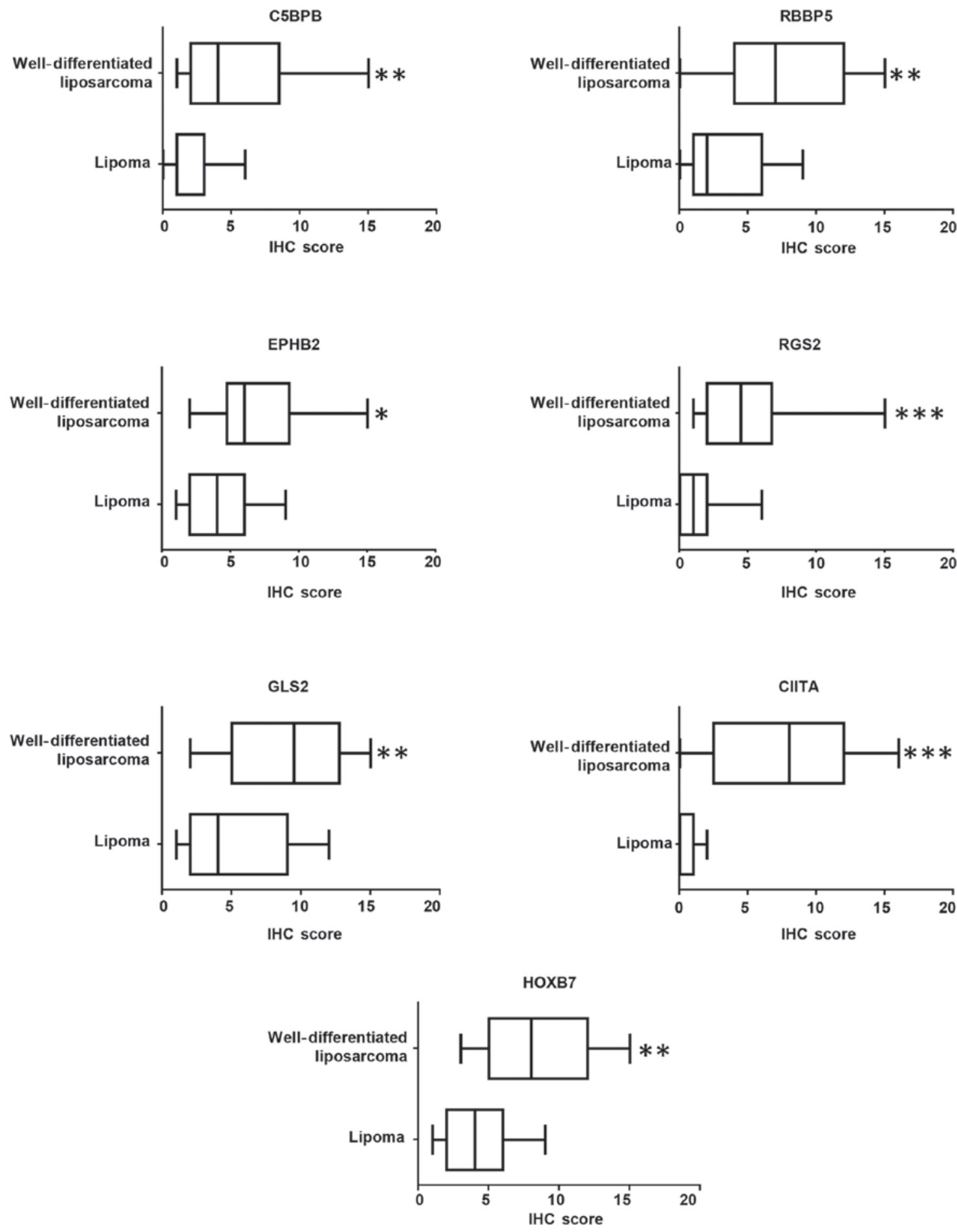

Figure 3. Distribution of diagnostic marker expression. Box and whisker plots indicate the distribution of protein expression for each antigen in the lipomatous tumor tissue panel. ${ }^{*} \mathrm{P} \leq 0.05,{ }^{* *} \mathrm{P} \leq 0.005$ and ${ }^{* * *} \mathrm{P} \leq 0.0005$.

tumor tissue is sparse and where the histological features are very similar. These issues lead to increased diagnostic costs because of the need to send uncertain biopsies to tertiary referral centers for correct classification $(20,21)$. A recent publication confirmed that MDM2 amplification detected by FISH, which is considered the gold standard for distinction of lipomas from well-differentiated liposarcomas, showed high concordance rates with tumor types when firm histological diagnoses were made and the tissues were not considered equivocal diagnoses (22). Despite this finding, the authors of that demonstrated that MDM2 amplification also occurs in other types of liposarcomas (22). Additionally, previous findings have shown that diverse soft tissue sarcomas harbor MDM2 amplifications in up to $40 \%$ of cases (23-26). MDM2 amplification occurs in $31.6 \%$ of 'possible' well-differentiated liposarcomas (22), in $28.6 \%$ of 'probably' well-differentiated liposarcomas (22), and in 5\% of large and deep-seated lipomas (18). Since FISH is both labor and cost intensive, 
development of more reliable and/or cost-effective diagnostic biomarkers for these lipomatous tumors may reduce cost and speed of diagnosis.

Various methods have been utilized to identify disease biomarkers, and within the past decade omics-based technologies have allowed the knowledge-guided computational identification of diagnostic and prognostic markers of disease characterization, progression, outcome, and treatment susceptibilities (27). Such studies have been previously employed for soft tissue sarcomas. For instance, bioinformatics analysis has been utilized on microarray data from diverse sets of soft tissue sarcomas revealing well-defined gene networks that may effectively classify sarcoma subtypes and serve as a useful tool for rational taxonomy and diagnosis of tumors (19,28-30). In this study, we performed a meta-analysis on a panel of lipomatous tumor samples that were previously classified based on gene expression analysis. We identified 301 genes whose mRNA expression differed at least 2-fold between lipomas and well-differentiated liposarcomas. Of these identified genes, we validated our findings at the protein level for 15 of them, finding that over half of the expression patterns identified at the mRNA level in our meta-analysis correlated well at the protein level. Indeed, our most reliable multiprotein model exhibited sensitivity at $83.3 \%$ and specificity at $90.9 \%$ with regard to differentiating lipomas from well-differentiated liposarcoma tumors.

\section{References}

1. Nguyen T and Zuniga R: Skin conditions: benign nodular skin lesions. FP Essent 407: 24-30, 2013.

2. Bancroft LW, Kransdorf MJ, Peterson JJ and O'Connor MI: Benign fatty tumors: classification, clinical course, imaging appearance, and treatment. Skeletal Radiol 35: 719-733, 2006.

3. Paunipagar BK, Griffith JF, Rasalkar DD, Chow LTC, Kumta SM and Ahuja A: Ultrasound features of deep-seated lipomas. Insights into Imaging 1: 149-153, 2010.

4. Miao C, Liu D, Zhang F, Wang Y, Zhang Y, Yu J, Zhang Z, Liu G, Li B, Liu X and Luo C: Association of FPGS genetic polymorphisms with primary retroperitoneal liposarcoma. Sci Rep 5: 9079, 2015

5. Zagars GK, Goswitz MS and Pollack A: Liposarcoma: outcome and prognostic factors following conservation surgery and radiation therapy. Int J Radiat Oncol Biol Phys 36: 311-319, 1996.

6. Clay MR, Martinez AP, Weiss SW and Edgar MA: MDM2 amplification in problematic lipomatous tumors: Analysis of FISH testing criteria. Am J Surg Pathol 39: 1433-1439, 2015.

7. Dei Tos AP: Liposarcoma: New entities and evolving concepts. Ann Diagn Pathol 4: 252-266, 2000.

8. Brisson M, Kashima T, Delaney D, Tirabosco R, Clarke A, Cro S, Flanagan AM and O'Donnell P: MRI characteristics of lipoma and atypical lipomatous tumor/well-differentiated liposarcoma: Retrospective comparison with histology and MDM2 gene amplification. Skeletal Radiol 42: 635-647, 2013.

9. Gaskin CM and Helms CA: Lipomas, lipoma variants, and well-differentiated liposarcomas (atypical lipomas): Results of MRI evaluations of 126 consecutive fatty masses. AJR Am J Roentgenol 182: 733-739, 2004.

10. O'Donnell PW, Griffin AM, Eward WC, Sternheim A, White LM, Wunder JS and Ferguson PC: Can experienced observers differentiate between lipoma and well-differentiated liposarcoma using only MRI? Sarcoma 2013: 982784, 2013.

11. Hasegawa T, Yamamoto S, Nojima T, Hirose T, Nikaido T, Yamashiro K and Matsuno Y: Validity and reproducibility of histologic diagnosis and grading for adult soft-tissue sarcomas. Hum Pathol 33: 111-115, 2002.

12. Nilbert M, Rydholm A, Mitelman F, Meltzer PS and Mandahl N: Characterization of the 12q13-15 amplicon in soft tissue tumors. Cancer Genet Cytogenet 83: 32-36, 1995.
13. Pilotti S, Della Torre G, Lavarino C, Di Palma S, Sozzi G, Minoletti F, Rao S, Pasquini G, Azzarelli A, Rilke F, et al: Distinct mdm2/p53 expression patterns in liposarcoma subgroups: Implications for different pathogenetic mechanisms. J Pathol 181: 14-24, 1997.

14. Shimada S, Ishizawa T, Ishizawa K, Matsumura T, Hasegawa $T$ and Hirose T: The value of MDM2 and CDK4 amplification levels using real-time polymerase chain reaction for the differential diagnosis of liposarcomas and their histologic mimickers. Hum Pathol 37: 1123-1129, 2006.

15. Sirvent N, Coindre JM, Maire G, Hostein I, Keslair F, Guillou L, Ranchere-Vince D, Terrier P and Pedeutour F: Detection of MDM2-CDK4 amplification by fluorescence in situ hybridization in 200 paraffin-embedded tumor samples: Utility in diagnosing adipocytic lesions and comparison with immunohistochemistry and real-time PCR. Am J Surg Pathol 31: 1476-1489, 2007.

16. Sozzi G, Minoletti F, Miozzo M, Sard L, Musso K, Azzarelli A, Pierotti MA and Pilotti S: Relevance of cytogenetic and fluorescent in situ hybridization analyses in the clinical assessment of soft tissue sarcoma. Hum Pathol 28: 134-142, 1997.

17. Weiss SW: Lipomatous tumors. Monogr Pathol 38: 207-239, 1996.

18. Wong DD, Low IC, Peverall J, Robbins PD, Spagnolo DV, Nairn R, Carey-Smith RL and Wood D: MDM2/CDK4 gene amplification in large/deep-seated 'lipomas': Incidence, predictors and clinical significance. Pathology 48: 203-209, 2016.

19. Nakayama R, Nemoto T, Takahashi H, Ohta T, Kawai A, Seki K, Yoshida T, Toyama Y, Ichikawa H and Hasegawa T: Gene expression analysis of soft tissue sarcomas: Characterization and reclassification of malignant fibrous histiocytoma. Mod Pathol 20: 749-759, 2007.

20. ArbiserZK, Folpe AL and Weiss SW: Consultative (expert) second opinions in soft tissue pathology. Analysis of problem-prone diagnostic situations. Am J Clin Pathol 116: 473-476, 2001.

21. Thway K and Fisher C: Histopathological diagnostic discrepancies in soft tissue tumours referred to a specialist centre. Sarcoma 2009: 741975, 2009.

22. Thway K, Wang J, Swansbury J, Min T and Fisher C: Fluorescence in situ hybridization for MDM2 amplification as a routine ancillary diagnostic tool for suspected well-differentiated and dedifferentiated liposarcomas: Experience at a tertiary center. Sarcoma 2015: 812089, 2015.

23. Kimura H, Dobashi Y, Nojima T, Nakamura H, Yamamoto N, Tsuchiya H, Ikeda H, Sawada-Kitamura S, Oyama T and Ooi A: Utility of fluorescence in situ hybridization to detect MDM2 amplification in liposarcomas and their morphological mimics. Int J Clin Exp Pathol 6: 1306-1316, 2013.

24. Miura Y, Keira Y, Ogino J, Nakanishi K, Noguchi H, Inoue T and Hasegawa T: Detection of specific genetic abnormalities by fluorescence in situ hybridization in soft tissue tumors. Pathol Int 62: 16-27, 2012.

25. Nakayama T, Toguchida J, Wadayama B, Kanoe H, Kotoura Y and Sasaki MS: MDM2 gene amplification in bone and soft-tissue tumors: Association with tumor progression in differentiated adipose-tissue tumors. Int J Cancer 64: 342-346, 1995.

26. Weaver J, Downs-Kelly E, Goldblum JR, Turner S, Kulkarni S, Tubbs RR, Rubin BP and Skacel M: Fluorescence in situ hybridization for MDM2 gene amplification as a diagnostic tool in lipomatous neoplasms. Mod Pathol 21: 943-949, 2008.

27. Diamandis EP. Present and future of cancer biomarkers. Clin Chem Lab Med 52: 791-794, 2014.

28. Hadj-Hamou NS, Laé M, Almeida A, de la Grange P, Kirova Y, Sastre-Garau X and Malfoy B: A transcriptome signature of endothelial lymphatic cells coexists with the chronic oxidative stress signature in radiation-induced post-radiotherapy breast angiosarcomas. Carcinogenesis 33: 1399-1405, 2012.

29. Skubitz KM and Skubitz AP: Characterization of sarcomas by means of gene expression. J Lab Clin Med 144: 78-91, 2004.

30. Tran D, VermaK, Ward K, DiazD, Kataria E, Torabi A, Almeida A, Malfoy B, Stratford EW, Mitchell DC, et al: Functional genomics analysis reveals a MYC signature associated with a poor clinical prognosis in liposarcomas. Am J Pathol 185: 717-728, 2015. 